higher cost and less than optimum management of infectious diseases.

Objectives To determine the relative likelihood of true allergy in patients suspected to have a penicillin allergy and to investigate the risk factors involved. We hypothesized that the vast majority of self-reported penicillin allergies are less likely to be true allergies when proper immunological work up is performed.

Methods Paediatric patients aged $0-18$ years presenting to the ADR clinic at the Children Hospital of Western Ontario (CHWO) with suspected antibiotic allergies were included. A retrospective review of charts was conducted to obtain demographic information and results from allergological and in vitro testing. Subjects were evaluated with a radioallergosorbent test (RAST) or the lymphocyte toxicity assay (LTA)/the in vitro platelet toxicity assay (iPTA) depending on whether the history was most consistent with an immediate allergy or a delayed hypersensitivity, respectively. Patients with negative RAST or LTA/iPTA were recommended to undergo confirmatory oral challenge test (OCT).

Results Ninety subjects were identified including 75 with possible penicillin allergy and 10 with suspected allergy to a nonpenicillin antibiotic. Five subjects presented with a mixed allergy. Based on the results from RAST, in vitro testing and OCTs, the prevalence of a true allergy in the penicillin group was $6.25 \%$ vs. $66.67 \%$ in the non-penicillin group $(p<$ 0.001). Patients presenting with severe reactions were more likely to be truly allergic $(p<0.01)$. In-patients were more likely to present with non-penicillin allergies and were subsequently more likely to have a true allergy $(\mathrm{p}<0.001)$.

Conclusions True allergy is very rare in patients with suspected penicillin allergies and can be determined with a proper work-up including OCT. Shorter protocols for the evaluation of these patients would be beneficial.

Disclosure(s) Nothing to disclose

\section{FETAL OUTCOME FOLLOWING DYDROGESTERONE EXPOSURE IN PREGNANCY}

G Koren*, D Gilboa. Maccabi Research Institute, Tel Aviv

\subsection{6/archdischild-2019-esdppp.4}

Background The progestin dydrogesterone (DYD) is widely used for threatened and recurrent miscarriages, as well as for dysfunctional bleeding, infertility and other obstetric and gynecological indications. While its apparent efficacy has been compared to other progestins, its fetal safety has not been investigated.

Objectives To follow up fetal outcome after gestational exposure to DYD.

Patients and methods Using a 2.5 million patients' database, we compared congenital malformations among babies exposed in utero to DYD between 1999 and 2016, to a control group not receiving this medication. We adjusted for concomitant exposure to in vitro fertilization (IVF) and to other forms of assisted reproductive technology (ART).

Results There were 8508 children exposed in uteo to DYD (4417 males, 4091 females) out of 777,422 live births. After excluding cases with concomitant exposure to IVF and other forms of ART, DYD was associated with increased risk for hypospadias [OR 1.28(95\% confidence interval 1.06-1.55)], overall cardiovascular malformations [OR 1.18 (1.06-1.33)], spina bifida [OR 2.29(1.32-3.97] and hydrpcephalus [OR 2.04
(1.28-3.25]. In additional analysis, including also those exposed to IVF and other forms of ART, there was also increased risk for cryptorchidism [1.37(1.19-1.58)] and congenital dislocation of the hip [OR 1.58(1.42-1.78)].

Conclusions DYD confers teratogenic effects after exposure to the recommended doses in pregnant women. Some of these adverse fetal effects are further augmented by concomitant use of IVF and other forms of ART. These independent teratogenic effects may have important implication for the child and family. Disclosure(s) Nothing to disclose

\section{PHYSIOLOGICALLY BASED PHARMACOKINETIC MODELLING TO CHARACTERIZE ACETAMINOPHEN PHARMACOKINETICS AND NAPQI FORMATION IN NON-PREGNANT AND PREGNANT WOMEN}

\begin{abstract}
${ }^{1,2} \mathrm{P}$ Mian ${ }^{* *}, 3,4,5 \mathrm{JN}$ van den Anker, ${ }^{6,7} \mathrm{~K}$ van Calsteren, ${ }^{8} \mathrm{P}$ Annaert, ${ }^{1} \mathrm{D}$ Tibboel, ${ }^{2,9} \mathrm{M}$ Pfister,

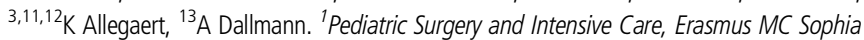
Children's Hospital, Rotterdam, The Netherlands; '2Pediatric Pharmacology and Pharmacometrics Research Center, University Children's Hospital Basel, Basel, Switzerland; ${ }^{3}$ Pediatric Surgery and Intensive Care, Erasmus Medical Center-Sophia Children's Hospital, Rotterdam, The Netherlands; ${ }^{4}$ Pediatric Pharmacology and Pharmacometrics Research Center, University Children's Hospital Basel (UKBB), Basel, Switzerland; ${ }^{5}$ Division of Pharmacology, Children's National Health System, Washington DC, DC, USA; ${ }^{6}$ Department of Obstetrics and Gynecology; ${ }^{7}$ Department of Development and Regeneration, University Hospitals Leuven; ${ }^{8}$ Drug Delivery and Disposition, KU Leuven, Leuven, Belgium; ${ }^{9}$ Pediatric Pharmacology and Pharmacometrics Research Center, University Children's Hospital Basel (UKBB), Basel, Switzerland; ${ }^{10}$ Certara LP, Princeton, NJ, United States; ${ }^{11}$ Department of Development and Regeneration, KU Leuven, Leuven, Belgium; ${ }^{12}$ Department of Pediatrics, Division of Neonatology, Erasmus Medical Center-Sophia Children's Hospital, Rotterdam, The Netherlands; ${ }^{13}$ Pediatric Pharmacology and Pharmacometrics Research Center, University Children's Hospital Basel (UKBB), Basel, Switzerland
\end{abstract}

\subsection{6/archdischild-2019-esdppp.5}

Background Little is known about the pharmacokinetics (PK) of acetaminophen during different stages of pregnancy. The aim of this study was to develop a physiologically based pharmacokinetic (PBPK) model to predict acetaminophen PK throughout pregnancy.

Methods PBPK models for acetaminophen and its metabolites were developed in non-pregnant and pregnant women. Physiological and enzymatic changes in pregnant women expected to impact acetaminophen PK were considered. The models were evaluated using goodness-of-fit-plots and through comparison of predicted PK profiles with in-vivo PK data. Predictions were performed to illustrate the concentrations at steady state $\left(\mathrm{C}_{\mathrm{ss}-\mathrm{mean}}\right)$, used as indicator for efficacy of acetaminophen achieved following $1000 \mathrm{mg}$ q6h. Furthermore, as measurement for potential hepatotoxicity, the molar dose fraction of acetaminophen converted to NAPQI was estimated.

Results PBPK models successfully predicted the PK of acetaminophen and its metabolites in populations of non-pregnant and pregnant women. Predictions resulted in lowest $\mathrm{C}_{\mathrm{ss}-\text { mean }}$ in the third trimester $(4.5 \mathrm{mg} / \mathrm{L})$, while $\mathrm{C}_{\mathrm{ss} \text {-mean }}$ was 6.7, 5.6 and $4.9 \mathrm{mg} / \mathrm{L}$ in non-pregnant, first and second trimester populations, respectively. Assuming a constant increased activity of CYP2E1 throughout pregnancy, the molar dose fraction of acetaminophen converted to NAPQI was highest during the first $(11.0 \%)$, followed by second $(9.0 \%)$ and third trimester $(8.2 \%)$, compared to non-pregnant women $(7.1 \%)$.

Conclusion Risk for drug related hepatotoxicity in pregnant women might be increased as more NAPQI is produced during pregnancy compared to non-pregnant women, especially 


\section{Expression of concern: 004 Fetal outcome following dydrogesterone exposure in pregnancy}

Koren G, Gilboa D. O04 Fetal Outcome following Dydrogesterone Exposure in Pregnancy. Arch Dis Child 2019;104:e2. doi: 10.1136/archdischild-2019-esdppp.4

The Editor-in-Chief of Archives of Disease in Childhood has been advised that the full report of this trial, published in Clinical Drug Investigation (https://doi.org/10.1007/s40261-019. 00862-w), has been retracted.

We do not have sufficient information about the reasons for retraction of the trial to provide evidence that this abstract is unreliable and should be retracted.

(C) Author(s) (or their employer(s)) 2021. No commercial re-use. See rights and permissions. Published by BMJ.

Arch Dis Child 2021;106:e2. doi:10.1136/archdischild-2019-esdppp.4eoc1

D) Check for updates 